\title{
Performance Analysis of ZF and MMSE channel equalizer for Butterworth and Chebyshev Low Pass Channels
}

\author{
Saurabh Shah, Jaymin Bhalani
}

\begin{abstract}
Due to the high demand for information in the communication system needs a greater amount of data transmission on the current channels. The data flow rate on the existing channel is bounded because of the Inter Symbol Interference (ISI). To minimize the influence of ISI Channel equalizers are utilized. The Zero Forcing (ZF) \& Minimum Mean Square Error (MMSE) equalizer is used to compare the eye diagrams before and after the equalizer in this paper. It shows that the equalizer reduce the ISI effectively and achieves the suitable performance.
\end{abstract}

Keywords : Channel Equalization, Eye Diagram, ISI, ZF \& MMSE Equalizer.

\section{INTRODUCTION}

Advancement in the field of Digital Transmission leads to having a colossal effect on human culture. The predicament of the ISI points to arise the common appearance of multipath propagation which demands to be reviewed by channel equalization [1]. The requirement for huge data has enhanced the demand for various equalization techniques [2]-[3]. Channel equalization [4] is adopted to enhance the acquired signal characteristic in digital communication systems. In this paper, the ZF [5] \& MMSE equalizer are used to balance the multipath transmission channel by lessening the influence of ISI [6].

\section{CHANNEL EQUALIZATION}

The system which remunerates the Channel Distortion is described as Channel Equalizer. Information channels where the channel elements are unexplored or time-varying, optimum transmit including receive filters cannot be composed directly and these channels require an equalizer to counterbalance the ISI produced by the deformity in the channel. There are three types of equalization practices ordinarily used:

- Maximum Likelihood (ML) Sequence Detection Optimal, but Impractical.

- Linear Equalization - Suboptimal, but simple.

- Non Linear Equalization (DFE) - for severe ISI channels.

Revised Manuscript Received on February 10, 2020.

* Correspondence Author

*Prof. Saurabh Shah, PhD Scholar, C U Shah University and EC Department, Babaria Institute of Technology, Vadodara, Gujarat, India, shahsaurabh1979@gmail.com

Dr. Jaymin Bhalani, EC Department, Babaria Institute of Technology, Vadodara, Gujarat, India, jaymin188@gmail.com

(C) The Authors. Published by Blue Eyes Intelligence Engineering and Sciences Publication (BEIESP). This is an open access article under the CC BY-NC-ND license (http://creativecommons.org/licenses/by-nc-nd/4.0/)
Linear Equalizers are manageable to execute and profoundly effective in channels where ISI is not rigid (like the wire-line telephone channel). Most maximum linear equalizers are performed as a linear transversal filter, shown in Fig 1.

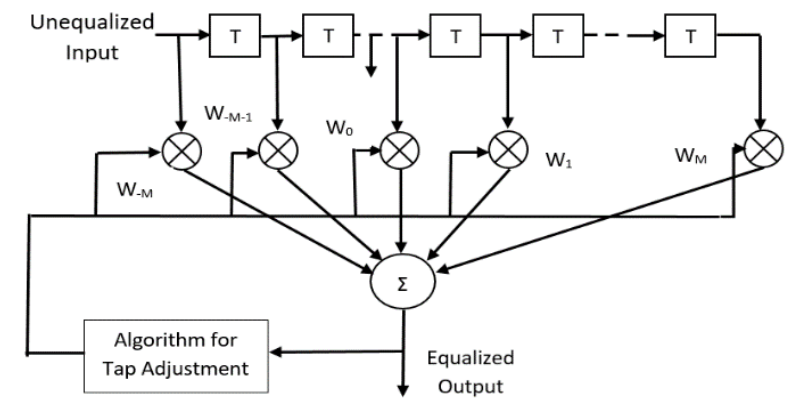

Fig 1. Linear Transversal Equalizer

Where $2 \mathrm{M}+1$ is the number of equalizer taps and the representation of duration is $\mathrm{T}$. If the input to the equalizer is $\mathrm{Y}(\mathrm{t})$, then the equalizer yield is given by,

$$
Y_{e q}(t)=\sum_{-M}^{i=M} w_{i} Y(t-i T)
$$

Based on a remarkable optimization the equalizer tap weights $\left(\mathrm{w}_{\mathrm{i}}\right)$ are chosen.

A. The Standard for Optimization: For optimizing the equalizer tap weights two patterns are ordinarily utilized:

- Peak Distortion Criterion - pointing to the Zero-Forcing Equalizer.

- Mean Square Error (MSE) Criterion - heading to the LMMSE equalizer and the Gradient (LMS) algorithm

B. Weight Adaptation: Linear Equalizers are further categorized into two classes based upon weight adaptation:

- Preset Equalizers: Worked for the channels where the frequency rejoinder properties are forgotten, but invariant (before-mentioned as the telephone channel). The weights are computed simply one (at the starting of the session) and not altered throughout the session.

- Adaptive Equalizers: Utilized by the time-variant frequency response channels. These Channels obtained the capacity to track the slow time-varying channels by regularly synchronizing the parameters.

\section{ZERO FORCING EQUALIZER}

The ZF equalizer refers to the collection of preset linear equalizers and it practices the Peak Distortion Criterion to judge the equalizer tap weights.

Suppose the block diagram with an equalizer for the communication system is in Fig 2. 


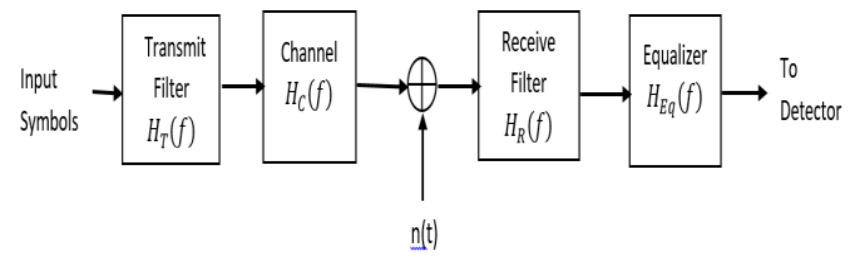

Fig 2. Communication System Block Diagram (with Equalizer)

Pretending that a raised-cosine pulse shape is employed, the situation for zero-ISI was given by,

$$
H_{T}(f) H_{C}(f) H_{R}(f) H_{E Q}(f)=H_{r c}(f)
$$

By description, $H_{T}$ (f) $H_{R}(f)=H_{r c}(f)$. Hence this $H_{E Q}(f)$ which counterbalances for the $\mathrm{H}_{\mathrm{C}}(\mathrm{f})$ channel distortion is given by,

$$
H_{E Q}(f)=\frac{1}{H_{C}(f)}
$$

This system means equalizer is named as an inverse channel equalizer. From equation (3),

$$
1=H_{E Q}(z) H_{C}(z)
$$

Or, in the discrete-time domain, we have,

$$
\sum_{i=-\infty}^{\infty} w_{j} h(n-j)=p_{e q}(n)= \begin{cases}1, & \text { if } n=0 \\ 0, & \text { if } n \neq 0\end{cases}
$$

Where, the impulse response of the channel is h(n) (discrete-time) and the response after equalization is $p_{e q}(t)$. Considering the filter which forces the ISI towards zero and because of this phenomenon it is described as Zero-Forcing Equalizer. Generally, ISI occurred due to the channel is bounded to a calculable number of symbols on both sides of the aspired symbols, it only needs to push the ISI towards zero just for these sampling moments. The effect of the traversal filter of finite duration (FIR) being displayed in Fig 1.

Derivation of the Filter Coefficients: The cascade of $\mathrm{H}_{\mathrm{T}}(\mathrm{f})$, $\mathrm{H}_{\mathrm{C}}(\mathrm{f}), \mathrm{H}_{\mathrm{R}}(\mathrm{f})$ plus the input (test) pulse $\mathrm{p}(\mathrm{t})$ corresponding to the unequalized pulse $\mathrm{p}_{\mathrm{r}}(\mathrm{t})$ into the channel. Suppose $2 \mathrm{M}+1$ is the period of each transversal filter. The equalized product pulse is yielded by,

$$
p_{\text {eq }}(t)=\sum_{i=-M}^{M} w_{i} p_{r}(t-i T)
$$

The ZF shape is immediately implemented on the specimens of $p_{e q}(t)$ accepted on sampling events $t=m T$.

$$
p_{\text {вq }}(m T)=\sum_{i=-M}^{M} w_{i} p_{r}((m-i) T)=\left\{\begin{array}{l}
1, \text { if } m=0 \\
0, \text { if } m=0, \pm 1, \pm 2, \ldots, \pm M
\end{array}\right.
$$

Which appears within a collection of $2 \mathrm{M}+1$ concurrent equations, whose clarification is provided through,

$$
\left(\begin{array}{c}
w_{-M} \\
\cdot \\
\cdot \\
w_{0} \\
\cdot \\
\cdot \\
w_{M}
\end{array}\right)=\left(\begin{array}{cccc}
p_{r}(0) & p_{r}(-1) & \cdots & p_{r}(-2 M) \\
p_{r}(1) & p_{r}(0) & \cdots & p_{r}(-2 M+1) \\
\cdot & \cdot & \cdot & \cdot \\
\cdot & \cdot & p_{r}(0) & p_{r}(-1) \\
p_{r}(2 M-1) & \ldots & p_{r}(1) & p_{r}(0) \\
p_{r}(2 M) & \cdots & &
\end{array}\right)^{-1}\left(\begin{array}{c}
0 \\
\cdot \\
\cdot \\
1 \\
\cdot \\
0
\end{array}\right)
$$

It is essential to note that the equalizer has a measurable length then employing the foregoing equation (8) does not remove the ISI fully. The residual or leftover ISI can be conquered by raising $\mathrm{M}$, while more extensive $\mathrm{M}$ will also enhance the complexity of designing as well as the implementation of the equalizer. As $\mathrm{M} \rightarrow \infty$, ISI is fully removed.

The additive noise is not counted by the Zero-Forcing Equalizer. Since the equalizer frequency reply is almost the inverse of the channel's frequency response (which is usually low pass), then the noise power at large frequencies into equalizer will cause a notable intensification. So, designed an equalizer with Noise and ISI both was taken into account will offer an enormously more trustworthy performance than the Zero-Forcing Equalizer.

\section{MINIMUM MEAN SQUARED ERROR EQUALIZER}

The Zero-Forcing Equalizer holds numerous setbacks because of its noise execution. The Linear Minimum Mean Squared Error (LMMSE) filter succeeds in this shortcoming by unbending the zero ISI condition and electing the equalizer characteristic such that the consolidated power in the ISI and the additive noise at the equalizer output is decreased.

Basic Assumption: The following are assumed in the derivation of the LMMSE equalizer.

- $E\left\{A_{n} A_{n+j}\right\}=\delta(j)$, means the input symbols are temporally uncorrelated.

- The input symbols are uncorrelated with noise.

The Derivation about the Equalizer Tap Weights: Consider the input to the equalizer,

$$
Y(n)=\sum_{k=-\infty}^{\infty} A_{k} p_{r}(n-k)+N(n)
$$

And the equalizer output,

$$
Y_{e q}(n)=\sum_{i=-M}^{M} w_{i} Y(n-i)
$$

The Equalizer product for the sampling times obtained by,

$$
Y_{\text {вq }}(n)=\sum_{i=-M}^{M} w_{i}\left[\sum_{k=-\infty}^{\infty} A_{k} p_{r}(n-i-k)+N(n-i)\right]
$$

The mean squared error (MSE) provided by,

$$
M S E=E\left\{\left(A_{n}-Y_{\text {eqq }}(n)\right)^{2}\right\}
$$

By applying every above-mentioned equation, the formation of the MSE can be produced by,

$M S E=E\left\{A_{n}^{2}\right\}-2 \sum_{i=-M}^{M} w_{i} E\left\{A_{n} Y(n-i)\right\}+E\left\{\left(\sum_{i=-M}^{M} w_{i} Y(n-i)\right)^{2}\right\}$

An additional solution of the earlier-mentioned equation is achievable through the subsequent events:

- $\mathrm{E}\left\{A_{n}^{2}\right\}=\sigma_{A}^{2}$ (by definition) 
- $E\left\{A_{n} Y(n-i)\right\}=\sigma_{A}^{2} \operatorname{pr}(-\mathrm{i})$ (applying the fundamental hypotheses)

- $E\{Y(n-i) Y(n-j)\}=\sigma_{A}^{2} \gamma(\mathrm{i}, \mathrm{j})+\sigma_{\mathrm{N}}^{2} \rho(\mathrm{i}-\mathrm{j})$ (applying the fundamental hypotheses) Where,

- $\gamma(\mathrm{i}, \quad \mathrm{j})=\sum_{l=-\infty}^{\infty} p_{r}(l-i) p_{r}(l-j)$ the auto-correlation of the unequalised pulse values.

- $\rho(\mathrm{i}-\mathrm{j})=E\{n(n-i) n(n-j)\}$, the auto-correlation of the receiver input noise.

- The Average Signal Power $\sigma_{A}^{2}$.

- The Average Noise Power $\sigma_{N}^{2}$.

By applying the given theories the earlier-mentioned equation (13) transformed into compact matrix notation:

- The received pulse values are $P_{r}=\left[p_{r}(M), \ldots, p_{r}(0), \ldots, p_{r}(-M)\right]^{T}$.

- $\Gamma$ is a matrix whose $(i, j)^{\text {th }}$ element is $\gamma(i, j)+\left(\sigma_{N}^{2} / \sigma_{A}^{2}\right) \rho(i-j)$.

- The equalizer tap-weights represents $\mathrm{w}=\left[\mathrm{w}-\mathrm{M}, \ldots, \mathrm{w}_{0}, \ldots, \mathrm{w}_{\mathrm{M}}\right]^{\mathrm{T}}$.

The final expression for the MSE is,

$$
M S E=\sigma_{A}^{2}\left(1-2 P_{r}^{T} w+w^{T} \Gamma w\right)
$$

The equation (14) differentiating concerning $\mathrm{w}_{\mathrm{i}}$, the equalizer tap weights and putting equal to zero, we get to the set of simultaneous equations and is given by,

$$
p_{r}=\Gamma w
$$

In equation (15) for uncorrelated (or white) input noise, the equation converts to

$$
p_{r}=\left(R+\frac{\sigma_{N}^{2}}{\sigma_{A}^{2}} I\right) w
$$

Where, the auto-correlation matrix of the unequalized pulse values is R plus an identity matrix is I. From the equation (16) the equalizer tap-weights can be estimated as:

$$
w=\left(R+\frac{\sigma_{N}^{2}}{\sigma_{A}^{2}} I\right)^{-1} p_{r}
$$

Understanding the fact that $\sigma_{\mathrm{A}}{ }^{2} / \sigma_{\mathrm{N}}{ }^{2}=\mathrm{SNR}$, we can rephrase the above equation (17) as:

$$
w=\left(R+\frac{1}{S N R} I\right)^{-1} p_{r}
$$

The compensation between the Noise and ISI is formulated by adopting the equation (18) for the designing of LMMSE equalizer. Each second term in all $\boldsymbol{\Gamma}$ matrix goes to zero if the receiver is producing an essentially noise-free condition $(\mathrm{SNR} \rightarrow \infty)$, and the solution resembles the zero-forcing equalizer solution. The noise expression is weighed properly in all the other cases.

After the equalization, the Residual mean squared error (MSE) can be figured using the expression,

$$
M S E=\sigma_{A}^{2}\left(1-P_{r}^{T} \Gamma_{p}\right)
$$

\section{SIMULATION}

A. ZF Equalizer: (1) The $4^{\text {th }}$ order Butterworth Low Pass Filter (LPF) used as a channel.

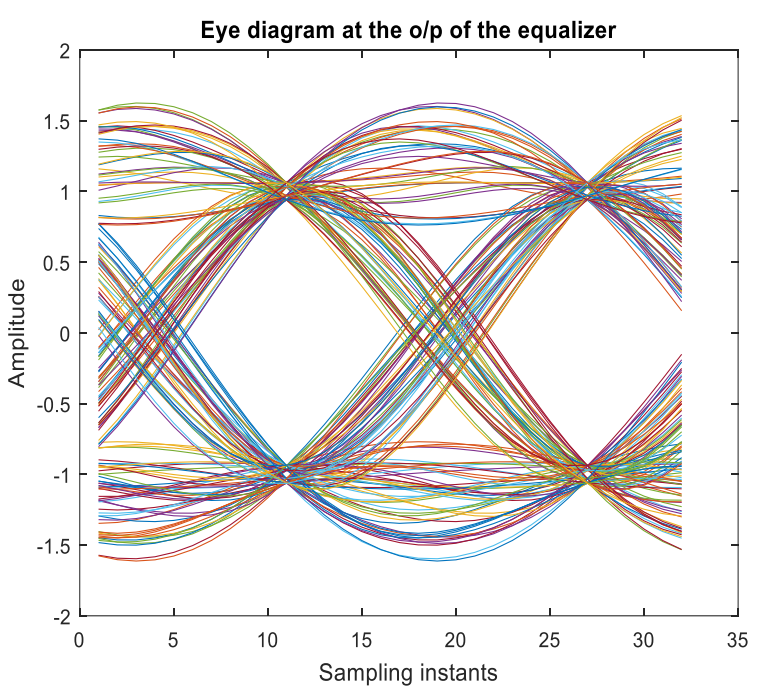

Fig 5. Eye Diagram at o/p of Equalizer

(2) The $4^{\text {th }}$ order Chebyshev LPF used as a channel. 


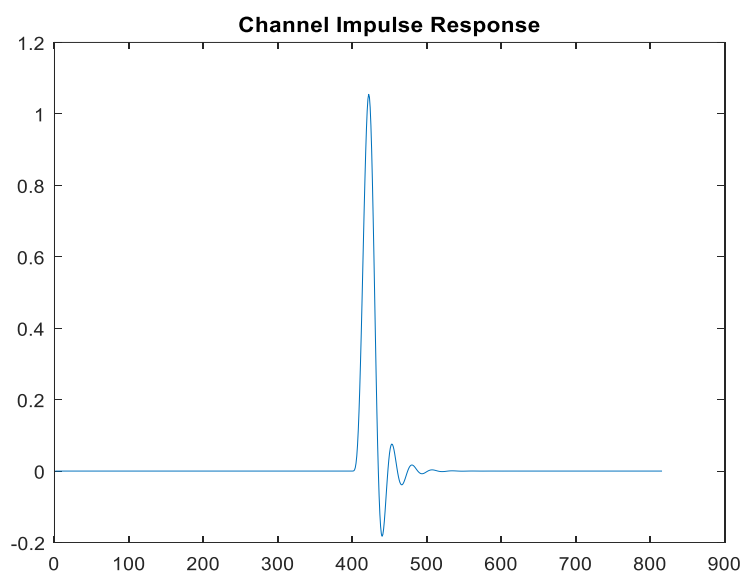

Fig 6. Impulse Response of Chebyshev LPF

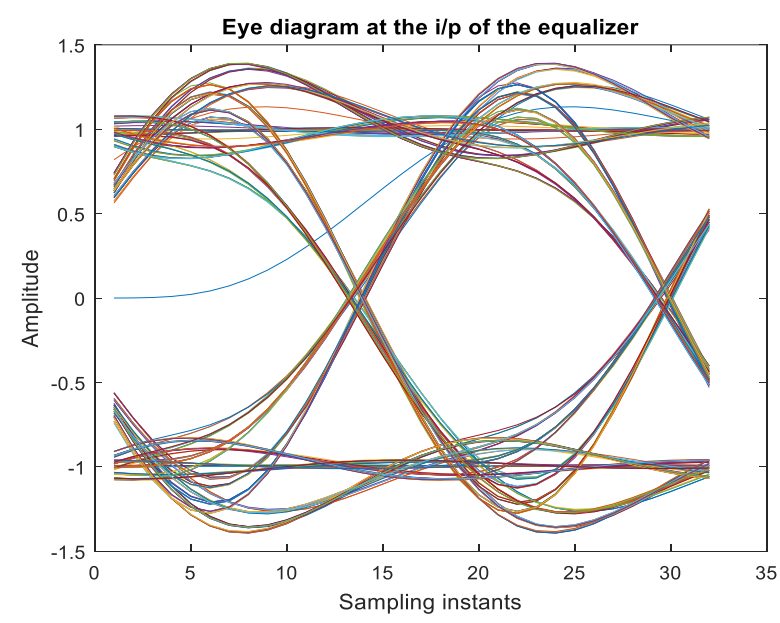

Fig 7. Eye Diagram at i/p of Equalizer

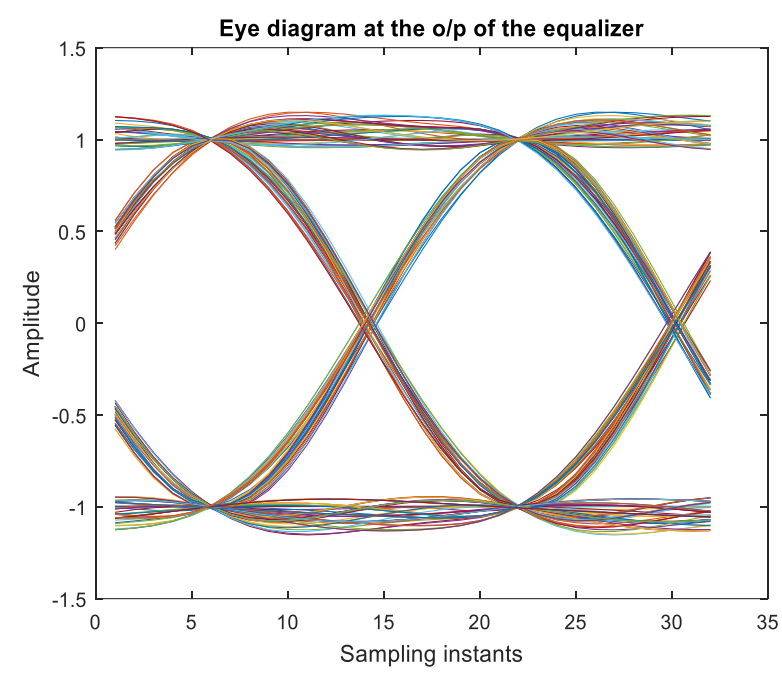

Fig 8. Eye Diagram at o/p of Equalizer

B. MMSE Equalizer: (1) The $4^{\text {th }}$ order Butterworth Low Pass Filter (LPF) used as a channel.

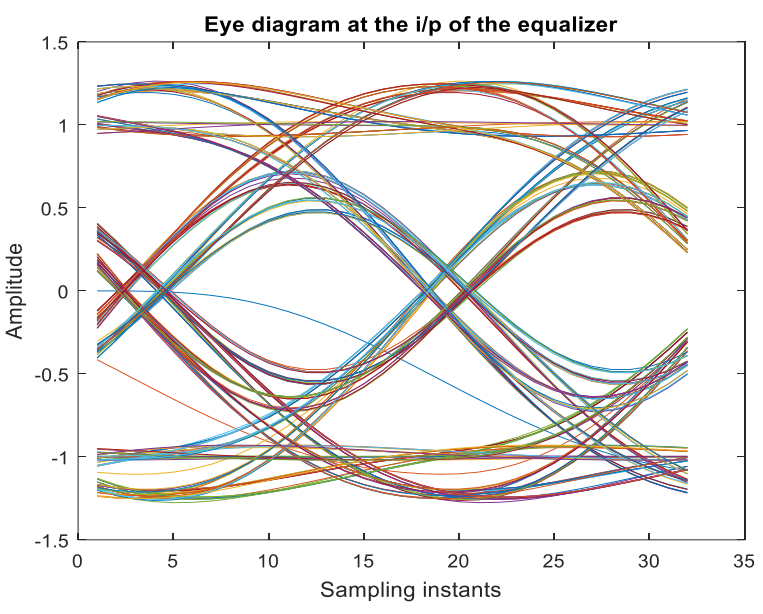

Fig 9. Eye Diagram at i/p of Equalizer

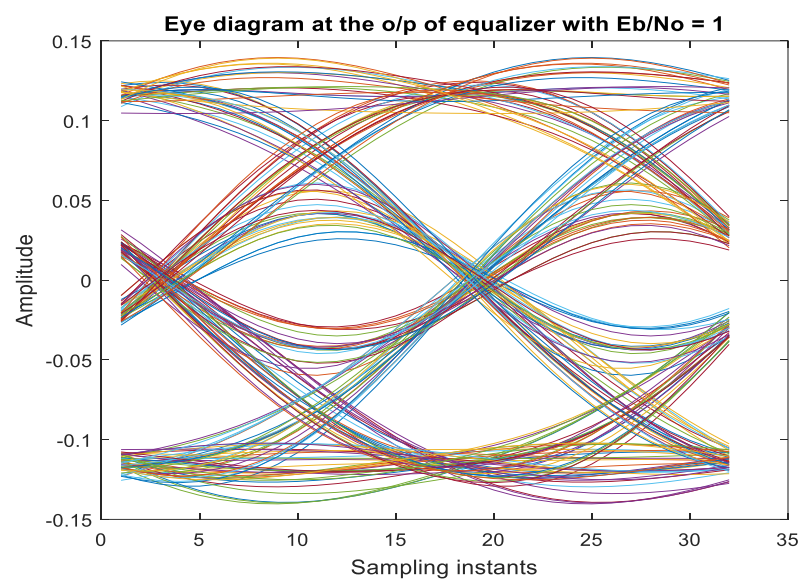

Fig 10. Eye Diagram at o/p of Equalizer with $\mathrm{Eb} / \mathrm{No}=1$

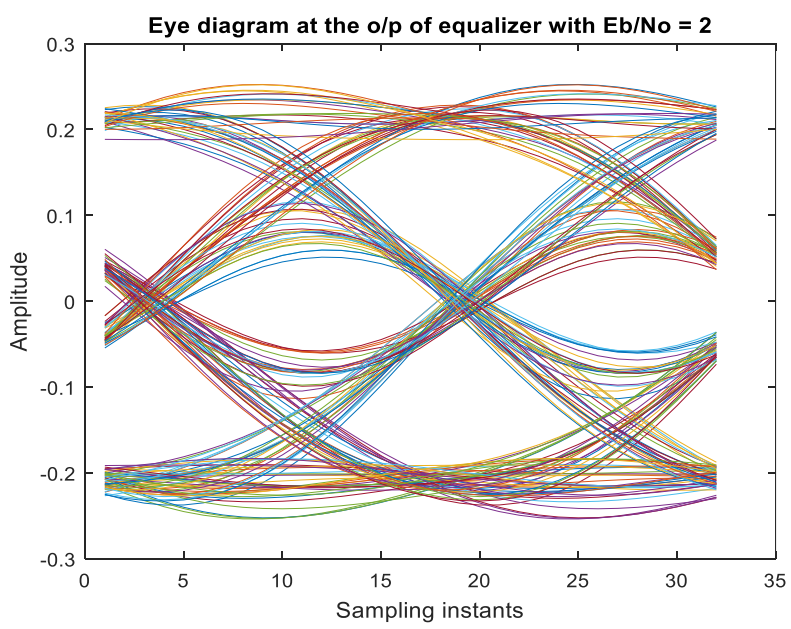

Fig 11. Eye Diagram at o/p of Equalizer with $\mathrm{Eb} / \mathrm{No}=2$ 


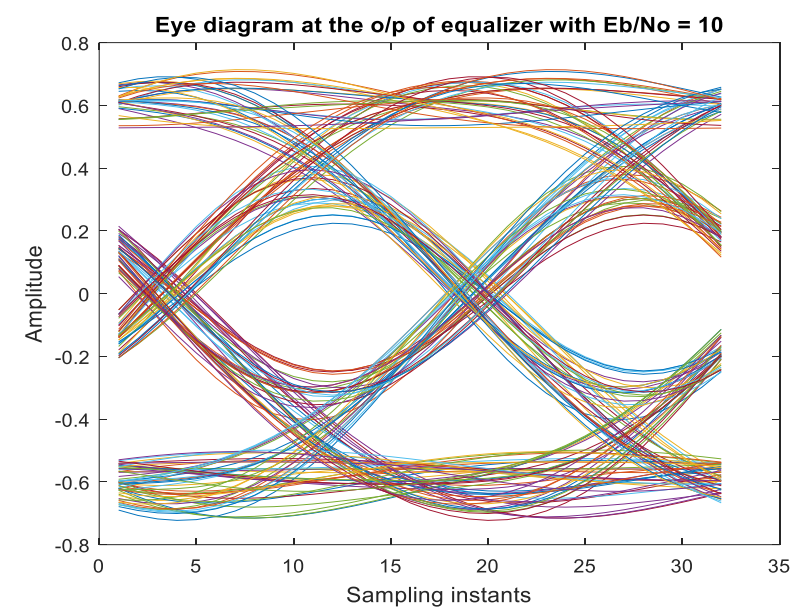

Fig 12. Eye Diagram at o/p of Equalizer with $\mathrm{Eb} / \mathrm{No}=10$

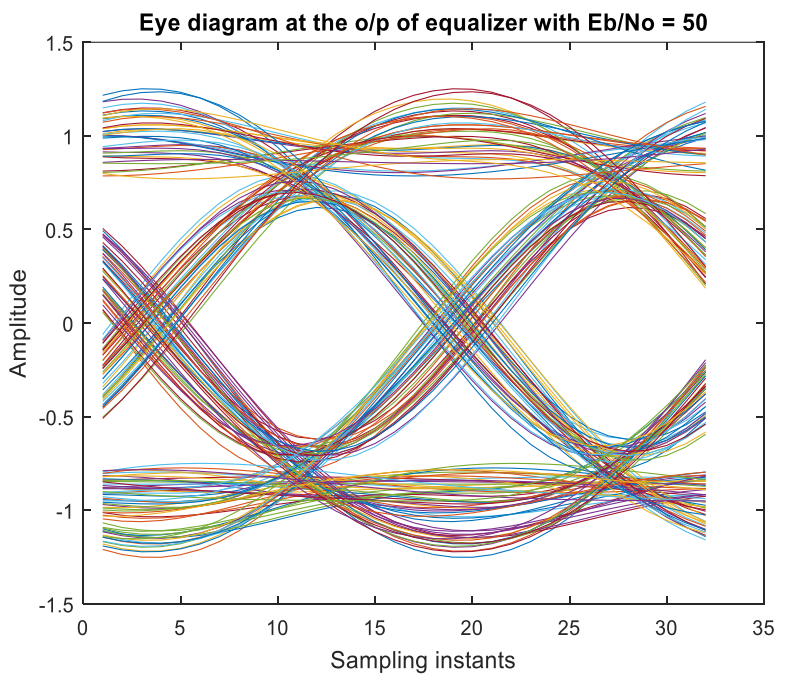

Fig 13. Eye Diagram at o/p of Equalizer with $\mathrm{Eb} / \mathrm{No}=50$

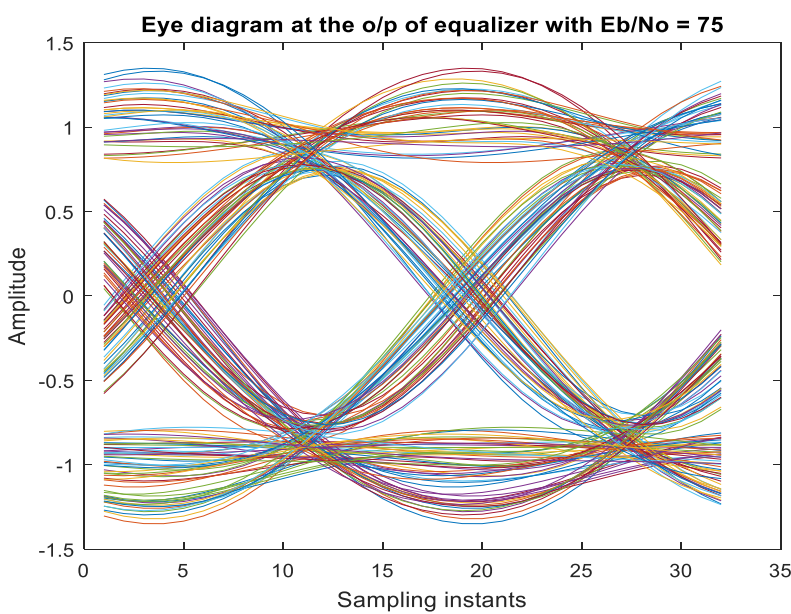

Fig 14. Eye Diagram at o/p of Equalizer with $\mathrm{Eb} / \mathrm{No}=75$

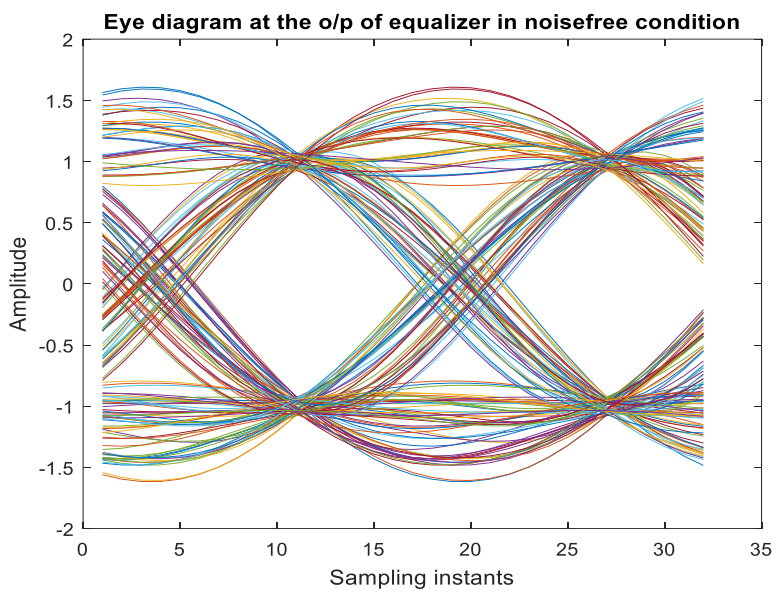

Fig 15. Eye Diagram at o/p of Equalizer in noise free condition

(2) The $4^{\text {th }}$ order Chebyshev LPF used as a channel.

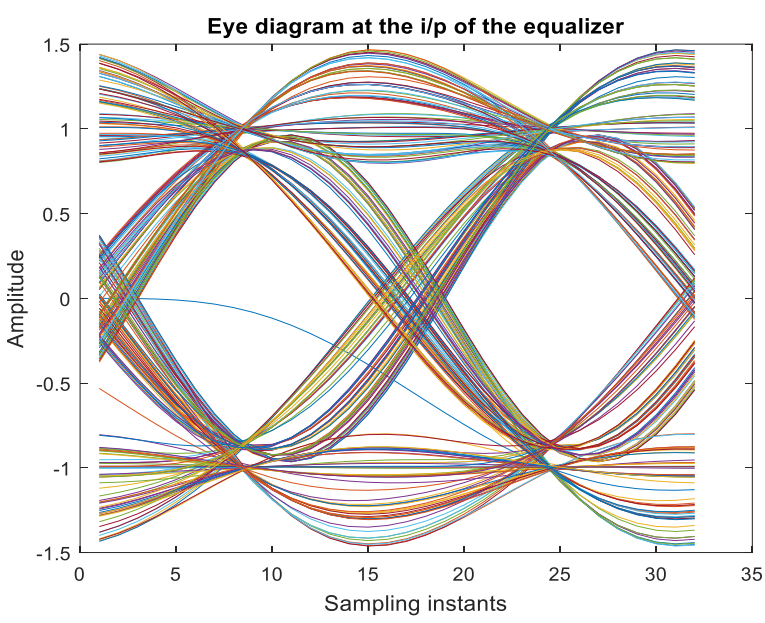

Fig 16. Eye Diagram at i/p of Equalizer

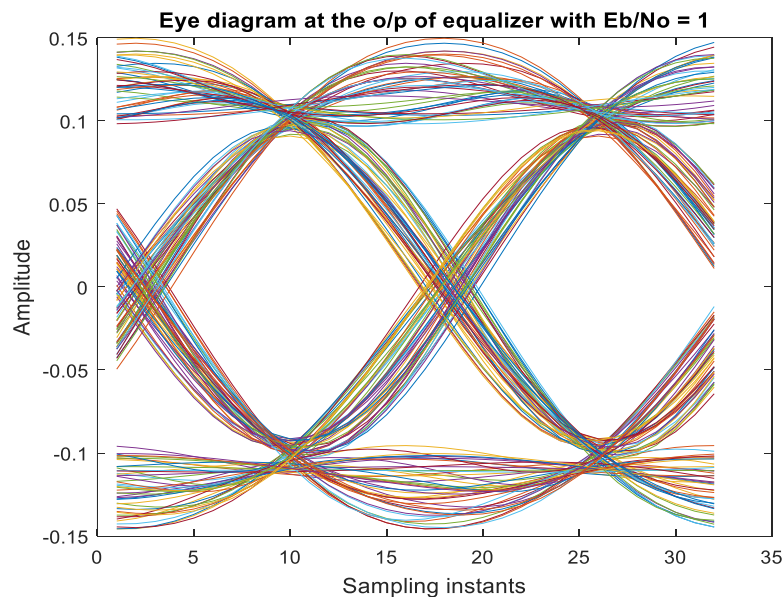

Fig 17. Eye Diagram at o/p of Equalizer with $\mathrm{Eb} / \mathrm{No}=1$

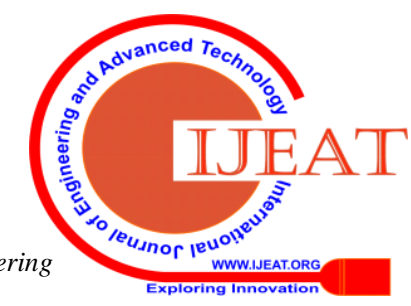




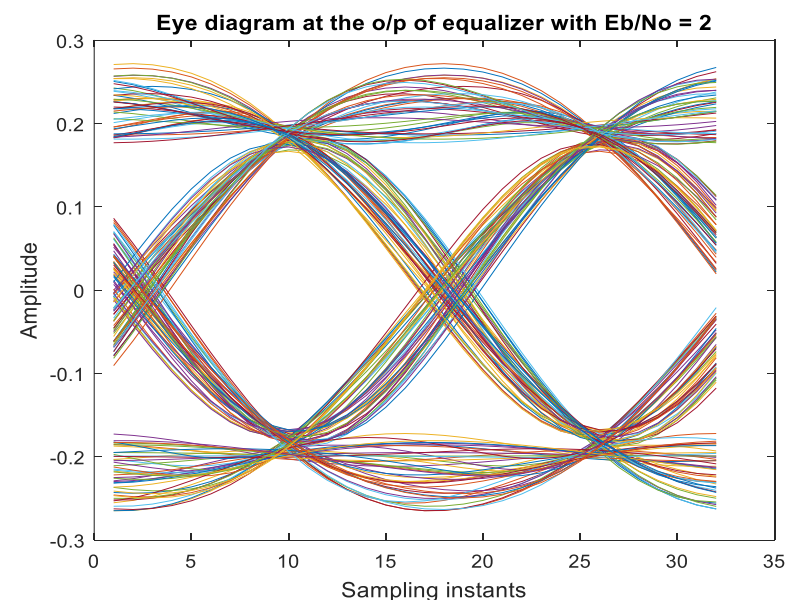

Fig 18. Eye Diagram at o/p of Equalizer with Eb/No $=2$

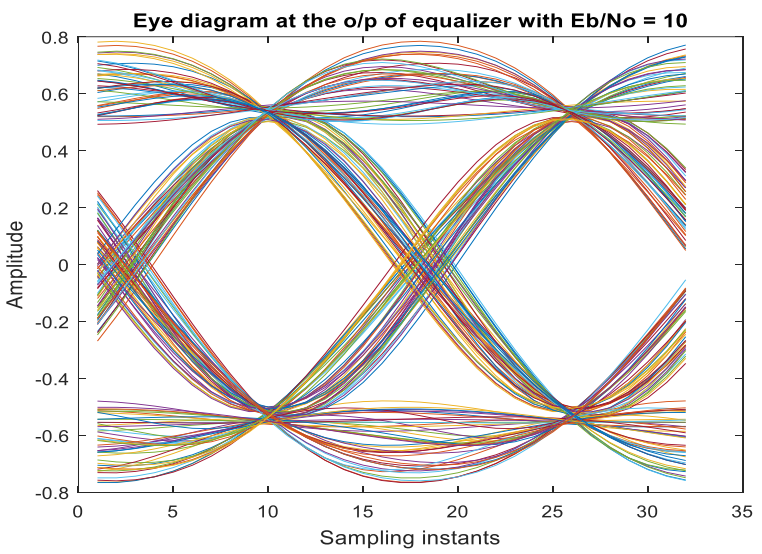

Fig 19. Eye Diagram at o/p of Equalizer with $\mathrm{Eb} / \mathrm{No}=10$

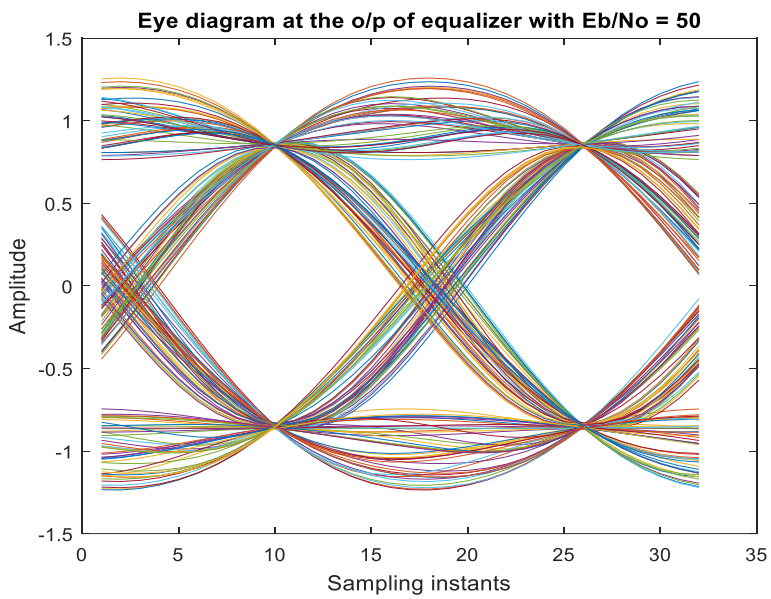

Fig 20. Eye Diagram at o/p of Equalizer with $\mathrm{Eb} / \mathrm{No}=\mathbf{5 0}$

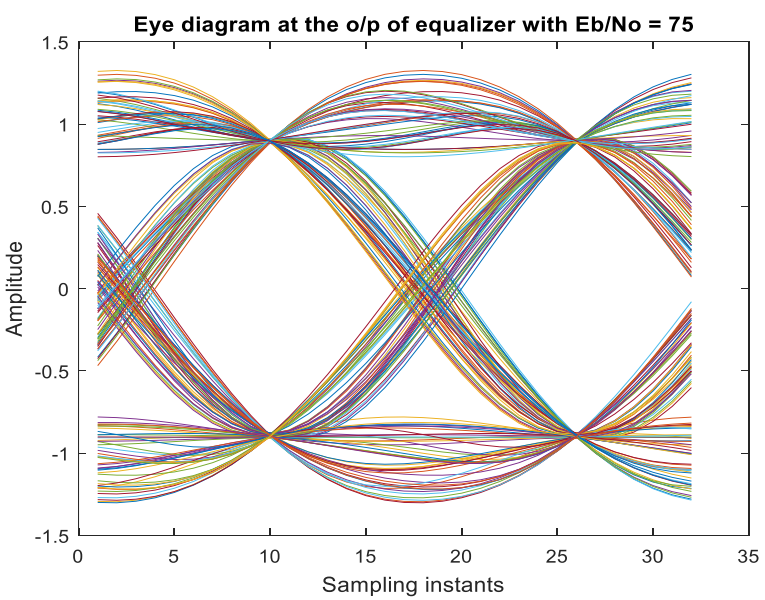

Fig 21. Eye Diagram at o/p of Equalizer with $\mathrm{Eb} / \mathrm{No}=75$

Retrieval Number: B4237129219/2020@BEIESP DOI: 10.35940/ijeat. B4237.029320

Journal Website: www.ijeat.org

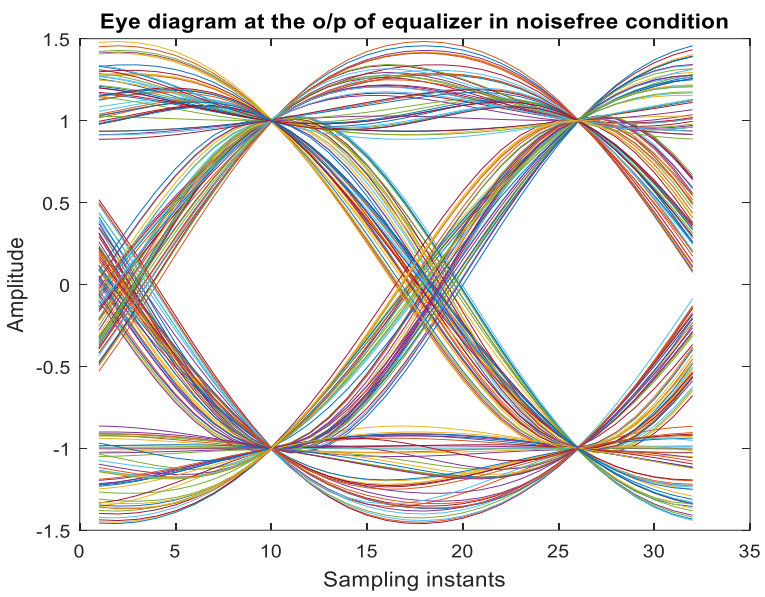

Fig 22. Eye Diagram at o/p of Equalizer in noise free condition

\section{RESULT}

\section{A. ZF Equalizer}

(1) The $4^{\text {th }}$ order Butterworth Low Pass Filter (LPF) used as a channel.

Table- I: Input \& Output MSE for Butterworth LPF

\begin{tabular}{|c|c|}
\hline & MSE \\
\hline Input & 0.0641 \\
\hline Output & 0.0023 \\
\hline
\end{tabular}

(2) The $4^{\text {th }}$ order Chebyshev LPF used as a channel.

Table- II: Input \& Output MSE for Chebyshev LPF

\begin{tabular}{|c|c|}
\hline & MSE \\
\hline Input & 0.0345 \\
\hline Output & $2.4792^{*} 10^{-5}$ \\
\hline
\end{tabular}

Simulation result depicts that for ZF equalizer mean Square error (MSE) Value before equalization is 0.0641 that improves after equalization is 0.0023 for the $4^{\text {th }}$ order Butterworth LPF. Same way, for $4^{\text {th }}$ order Chebyshev LPF, input value before equalization is 0.0345 that improves after equalization is $2.4792 * 10^{-5}$ that can be observed using eye diagram.

\section{B. MMSE Equalizer}

(1) The $4^{\text {th }}$ order Butterworth Low Pass Filter (LPF) used as a channel.

Table- III: MSE for Butterworth LPF

\begin{tabular}{|c|c|}
\hline Eb / No (dB) & MSE \\
\hline 1 & 0.9184 \\
\hline 2 & 0.8505 \\
\hline 10 & 0.5468 \\
\hline 50 & 0.2094 \\
\hline 75 & 0.1528 \\
\hline
\end{tabular}

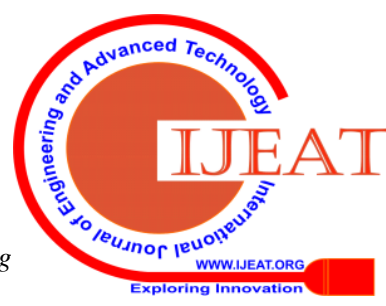




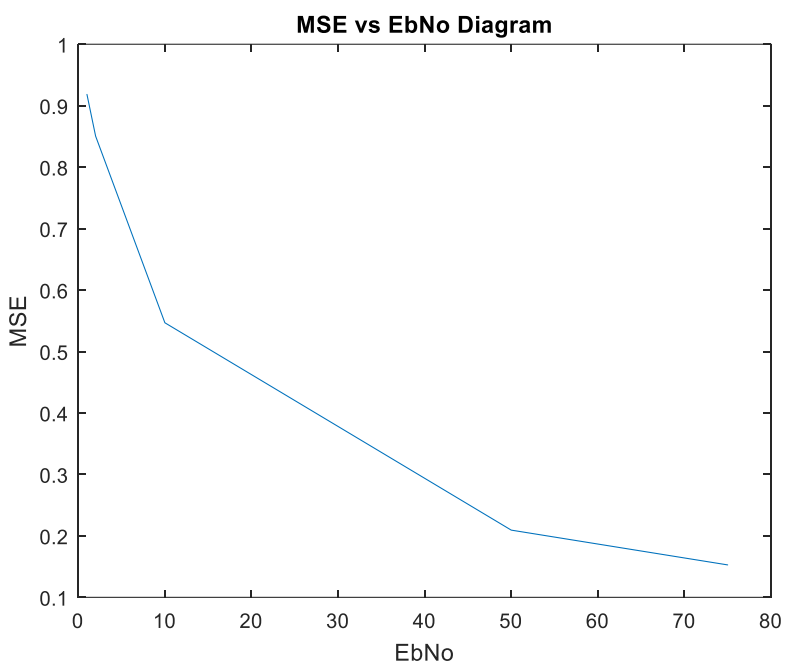

Fig 23. MSE v/s Eb/No Diagram

(2) The $4^{\text {th }}$ order Chebyshev LPF used as a channel.

Table- IV: MSE for Chebyshev LPF

\begin{tabular}{|c|c|}
\hline Eb / No $(\mathrm{dB})$ & MSE \\
\hline 1 & 0.8981 \\
\hline 2 & 0.8150 \\
\hline 10 & 0.4687 \\
\hline 50 & 0.1501 \\
\hline 75 & 0.1054 \\
\hline
\end{tabular}

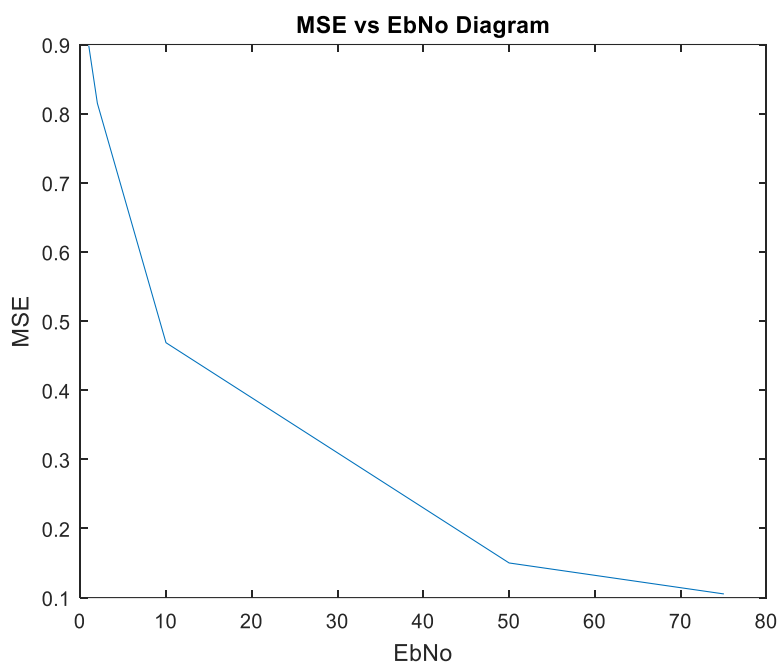

Fig 24. MSE v/s Eb/No Diagram

It can be observed from table that MSE value improves with increasing Eb/No values as noise decreases, for $10 \mathrm{~dB}$ MSE is 0.5468 for $4^{\text {th }}$ order Butterworth LPF which improves for $\mathrm{Eb} / \mathrm{No}$ equal to 75 for that MSE is 0.1528 . Same way for $4^{\text {th }}$ order Chebyshev, MSE improves as Eb/No increases. Result is described in Table- III \& IV herewith.

\section{CONCLUSION}

This paper depicts performance analysis of ZF and MMSE channel equalization techniques for $4^{\text {th }}$ order Butterworth low pass filter and Chebyshev LPF channels using various values of Eb/No for simulations. Results show that improvement in eye diagrams and mean square error (MSE) with respect to increase in Eb/No respectively.

\section{REFERENCES}

1. J. G. Proakis, Digital Communication, McGraw Hill, New York, 2001.

2. S. U. H. Qureshi, "Adaptive Equalization," IEEE, vol. 73, pp 1349-1387, September 1985.

3. S. Bhardwaj, "A Review on Adaptive Equalizer Algorithms", International Journal of Scientific Research, ISSN: 2277-8179, Vol 3, Issue 7, 2014.

4. N S Randhawa, "An overview of Adaptive Channel Equalization Techniques \& Algorithms", International Journal of Science \& Research, vol 3, Issue 7, July - 2014.

5. Prachi Sharma, "Performance Comparison of ZF, LMS \& RLS Algorithms for Linear Adaptive Equalizer", Advance in Electronic \& Electrical Engineering, ISSN: 2231-1297, Vol 4, Issue 6, 2014.

6. Linghui Wang \& Wei He, "Adaptive Channel Equalization based on RLS Algorithm", IEEE International conference on system science, Engineering Design \& Manufacturing Information, 2011.

\section{AUTHORS PROFILE}

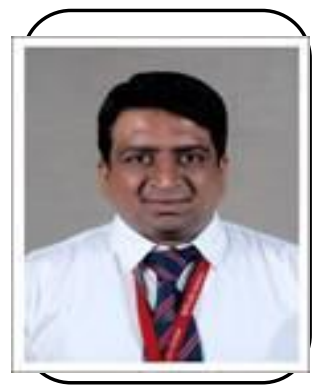

S. P. Shah received a B.Sc. degree in Mathematics from M S University, BE degree in Electronics Engineering from M S University and ME degree in Industrial Electronics from M S University, Baroda. He is currently working in E\&C dept., Babaria Institute of Technology, Varnama, Vadodara and also doing a Ph.D from C U Shah University, Wadhvan, Surendranagar. His area of interest is in Communication Systems, Signal Processing, Power Electronics, etc.

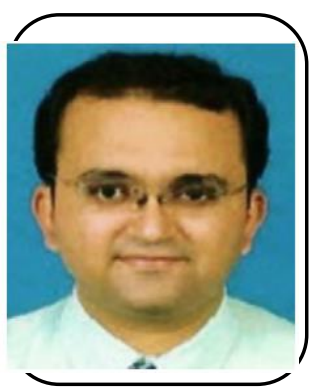

J. K. Bhalani received the $\mathrm{BE}$ degree in E \& C engineering from South Gujarat University, an M.E. degree in Communication System Engineering from Gujarat University and Ph.D. from Faculty of Technology, M S Univjersity of Baroda. He is currently working as Professor in E \& C dept., Babaria Institute of Technology, Baroda. His area of interest is in Communication Systems, MIMO Communication system, VLSI, Signal processing etc. 